\title{
Aberrant Hippocampal Activity Underlies the Dopamine Dysregulation in an Animal Model of Schizophrenia
}

\author{
Daniel J. Lodge and Anthony A. Grace \\ Departments of Neuroscience, Psychiatry, and Psychology, University of Pittsburgh, Pittsburgh, Pennsylvania 15260
}

\begin{abstract}
Evidence supports a dysregulation of subcortical dopamine (DA) system function as a common etiology of psychosis; however, the factors responsible for this aberrant DA system responsivity have not been delineated. Here, we demonstrate in an animal model of schizophrenia that a pathologically enhanced drive from the ventral hippocampus (vHipp) can result in aberrant dopamine neuron signaling. Adult rats in which development was disrupted by prenatal methylazoxymethanol acetate (MAM) administration display a significantly greater number of spontaneously firing ventral tegmental DA neurons. This appears to be a consequence of excessive hippocampal activity because, in MAM-treated rats, vHipp inactivation completely reversed the elevated DA neuron population activity and also normalized the augmented amphetamine-induced locomotor behavior. These data provide a direct link between hippocampal dysfunction and the hyper-responsivity of the DA system that is believed to underlie the augmented response to amphetamine in animal models and psychosis in schizophrenia patients.
\end{abstract}

Key words: hippocampus; schizophrenia; MAM; dopamine; psychosis; animal model

\section{Introduction}

The role of dopamine (DA) in schizophrenia is well established based on the ability of DA agonists to exacerbate psychosis, the efficacy of DA antagonists in treating schizophrenia, and imaging studies of increased amphetamine-induced DA release in schizophrenia (Laruelle and Abi-Dargham, 1999; Abi-Dargham, 2004). Nonetheless, there is no evidence for a primary pathology within the DA system itself in schizophrenia; rather, the DA system appears to be abnormally regulated (Grace, 1991, 2000; AbiDargham, 2004). However, the mode of this dysregulation is unknown. One model of DA function posits that the mesolimbic DA system is regulated via two independent mechanisms: (1) transient or "phasic" DA release driven by DA neuron burst firing and (2) extrasynaptic or "tonic" levels DA dependent on basal DA neuron activity and regulated via presynaptic inputs (Grace, 1991; Floresco et al., 2003). DA neuron burst firing induces a large transient increase in perisynaptic DA (Chergui et al., 1994) and is considered to be the functionally relevant signal that encodes reward prediction or incentive salience (Berridge and Robinson, 1998; Schultz, 1998), whereas tonic DA transmission occurs on a much slower scale and is proposed to set the background level of DA system activation (Grace, 1991).

DA neuron activity is potently modulated by the ventral hip-

Received June 22, 2007; revised Sept. 6, 2007; accepted Sept. 6, 2007.

This work was supported by United States Public Health Service Grants DA15408 and MH57440 (A.A.G.) and a Young Investigator Award from the National Alliance for Research on Schizophrenia and Depression, The Mental Health Research Association (D.J.L.). We thank Niki MacMurdo and Christy Smolak for their valuable assistance and Brian Lowry for the production, development, and support with the custom-designed electrophysiology software (Neuroscope).

Correspondence should be addressed to Daniel J. Lodge, Department of Neuroscience, University of Pittsburgh, A210 Langley Hall, Pittsburgh, PA 15260. E-mail: Lodge@bns.pitt.edu.

D01:10.1523/JNEUROSCI.2847-07.2007

Copyright $\odot 2007$ Society for Neuroscience $\quad 0270-6474 / 07 / 2711424-07 \$ 15.00 / 0$ pocampus (vHipp) (Legault and Wise, 1999; Floresco et al., 2003; Lodge and Grace, 2006a). Thus, vHipp activation increases DA neuron population activity (i.e., number of DA neurons firing spontaneously) without affecting average firing rate or burst firing and, moreover, this is dependent on a polysynaptic [vHippnucleus accumbens (NAc)-ventral pallidum (VP)] projection (Floresco et al., 2003; Lodge and Grace, 2006a). An increase in population activity makes the DA system more responsive to phasic activation by glutamatergic afferents (Lodge and Grace, 2006a). Given evidence correlating hippocampal dysfunction with psychosis in schizophrenia (Harrison, 2004), we propose that aberrant hippocampal activity may underlie the DA dysregulation in this disorder.

Previous attempts to produce an animal model of schizophrenia have relied on developmental disruption. One model using administration of the DNA methylating agent, methylazoxymethanol acetate (MAM) to pregnant dams on gestational day 17 (GD17) has substantial face validity in that the adult offspring demonstrate anatomical changes (thinning of limbic cortices with increased neuronal packing density) (Moore et al., 2006), behavioral deficits [decreased prepulse inhibition of startle, disruption in learning new response contingencies, increased responses to PCP (phencyclidine) and amphetamine (Flagstad et al., 2004; Moore et al., 2006), an increased sensitivity to stress (Goto and Grace, 2006), executive behavioral impairment (Gourevitch et al., 2004), perseverative errors and deficits in latent inhibition (Flagstad et al., 2004), and social impairment (Talamini et al., 1998; Talamini et al., 2000)], and disruption of rhythmic activity in frontal cortex (Goto and Grace, 2006) that parallels what has been observed in schizophrenia patients. Thus, the MAM GD17 model recapitulates a pathodevelopmental process leading to schizophrenia-like neuroanatomical and behavioral phenotypes. Using this model, we now demonstrate the 
presence of a hippocampal dysfunction that leads to DA system hyper-responsivity and provide new insights into understanding the role of these systems in the pathophysiology of schizophrenia.

\section{Materials and Methods}

All experiments were performed in accordance with the guidelines outlined in the United States Public Health Service Guide for the Care and Use of Laboratory Animals and were approved by the Institutional Animal Care and Use Committee of the University of Pittsburgh.

Animals. MAM treatments were performed as described previously (Moore et al., 2006). In brief, timed pregnant female Sprague Dawley rats were obtained at GD15 and housed individually in plastic breeding tubs. MAM (diluted in saline, $20 \mathrm{mg} / \mathrm{kg}$, i.p.) was administered on GD17. Control rats received injections of saline $(1 \mathrm{ml} / \mathrm{kg}$, i.p.). Within a week of birth, offspring were culled to 10 by removal of female rats. Male pups were weaned on day 21 and housed in groups of two to three with littermates until $\sim 4-5$ months of age, at which time they were used for physiological or behavioral studies. All experiments were performed on multiple litters of MAM- and saline-treated rats.

Acute studies. Adult male rats were anesthetized with chloral hydrate (400 mg/kg, i.p.) and placed in a stereotaxic apparatus. Anesthesia was maintained by supplemental administration of chloral hydrate as required to maintain suppression of limb compression withdrawal reflex and a core body temperature of $37^{\circ} \mathrm{C}$ was sustained by a thermostatically controlled heating pad. For acute administration of drugs into discrete brain areas, rats were implanted with 23 gauge cannulas $2.0 \mathrm{~mm}$ dorsal to the vHipp [anteroposterior (AP), -6.0 ; mediolateral $(\mathrm{ML}),+5.3$; dorsoventral (DV), $-4.5 \mathrm{~mm}$ from bregma] and the pedunculopontine tegmental nucleus (PPTg; AP, -8.0 ; ML, +1.6; DV, $-5.0 \mathrm{~mm}$ from bregma) that were fixed in place with dental cement and two anchor screws.

Pharmacological manipulations. Chemical stimulation was specifically used to enable neuronal excitation without the confounds associated with current spread, activation of fibers of passage, or potential lesions during extended stimulation that may occur with electrical activation. All drugs were dissolved in Dulbecco's PBS (dPBS) and infused in a volume of $0.5 \mu \mathrm{l}$ through a 30 gauge injection cannula protruding $2.0 \mathrm{~mm}$ past the end of the implanted guide cannula. The injection cannula was left in situ for 1-2 min to ensure diffusion of drug into the surrounding tissue. NMDA $(0.75 \mu \mathrm{g} / 0.5 \mu \mathrm{l})$ (Floresco et al., 2003; Lodge and Grace, 2006a), tetrodotoxin (TTX; $1 \mu \mathrm{M}$ in $0.5 \mu \mathrm{l}$ ) (Floresco et al., 2001), or vehicle (dPBS) were all injected at doses reported previously to induce specific behavioral and/or neurochemical effects.

The control group for DA neuron population studies consisted of rats that received either no injection or vehicle (dPBS) infusions into the vHipp and PPTg. Consistent with previous data (Lodge and Grace, 2006a) these groups all showed similar DA neuron population activity parameters and their data were combined. Rats received only one injection per region and DA cell recordings were typically recorded from 10 min to $2 \mathrm{~h}$ after infusions.

VTA DA neuron extracellular recordings. Extracellular microelectrodes (impedance, 6-14 M $\Omega$ ) were lowered into the ventral tegmental area (VTA; AP, -5.3 ; ML, $+0.8 \mathrm{~mm}$ from bregma and -6.5 to $-9.0 \mathrm{~mm}$ ventral of brain surface) using a hydraulic microdrive and the activity of the population of DA neurons was determined by counting the number of spontaneously active DA neurons encountered while making 5-9 vertical passes, separated by $200 \mu \mathrm{m}$, in a predetermined pattern to sample equivalent regions of the VTA. Spontaneously active DA neurons were identified with open filter settings (low pass, $50 \mathrm{~Hz}$; high pass, $16 \mathrm{kHz}$ ) using previously established electrophysiological criteria (Grace and Bunney, 1983) and, once isolated, their activity was recorded for 2-3 min. Three parameters of activity were measured: (1) population activity (defined as the number of spontaneously active DA neurons recorded per electrode track), (2) basal firing rate, and (3) the proportion of action potentials occurring in bursts (defined as the occurrence of two spikes with an interspike interval of $<80 \mathrm{~ms}$, and the termination of the burst defined as the occurrence of an interspike interval of $>160 \mathrm{~ms}$ ) (Grace and Bunney, 1983). $v$ Hipp extracellular recordings. Extracellular microelectrodes (impedance 6-12 M $\Omega$ ) were lowered into the ventral hippocampus (AP, -5.0; $\mathrm{ML},+4.5 \mathrm{~mm}$ from bregma and -5.0 to $-8.5 \mathrm{~mm}$ ventral of brain surface) using a hydraulic microdrive and spontaneously active neurons throughout the vHipp were recorded while making $8-12$ vertical passes (moving caudal and lateral), separated by $200 \mu \mathrm{m}$, in a predetermined pattern to sample equivalent regions of the vHipp. Once isolated, vHipp neuronal activity was recorded for $3 \mathrm{~min}$.

Amphetamine-induced locomotion. All survival surgical procedures were performed under general anesthesia in a semisterile environment. Briefly, male rats were anesthetized with ketamine/xylazine (80/12 mg/ $\mathrm{kg}$, i.p., respectively) and placed in a stereotaxic apparatus using blunt atraumatic ear bars. Bilateral cannulas (23 gauge) were implanted $2 \mathrm{~mm}$ dorsal to the ventral hippocampus (AP, -6.0 ; ML, \pm 5.3 ; DV,$-4.5 \mathrm{~mm}$ from bregma) and fixed in place with dental cement and four anchor screws. Once the cement was completely solid, the wound was sutured, the rat removed from the stereotaxic frame and monitored closely until conscious. Rats received antibiotic treatment (gentamicin $3 \mathrm{mg} / \mathrm{kg}$, s.c.) and postoperative analgesia (Children's Tylenol syrup in softened rat chow; 5\% v/w) ad libitum for $24 \mathrm{~h}$. Rats were housed with a reverse light/dark cycle (lights on from 7:00 P.M. to 7:00 A.M.) for at least 2 weeks before behavioral experiments. Rats were administered TTX (1 $\mu \mathrm{M})$ or vehicle (dPBS) bilaterally $(0.5 \mu \mathrm{l} /$ side $)$ and placed in an open field arena (Coulbourn Instruments, Allentown, PA) where spontaneous locomotor activity in the $x-y$ plane was determined for $30 \mathrm{~min}$ by beam breaks and recorded with TruScan software (Coulbourn Instruments). Rats were then injected with D-amphetamine sulfate $(0.5 \mathrm{mg} / \mathrm{kg}$, i.p. $)$ and locomotor activity recorded for an additional $60 \mathrm{~min}$. It should be noted that a subpopulation of rats were also tested for prepulse inhibition of startle for the purpose of another study.

Histology. At the cessation of the electrophysiology experiments, the recording site was marked via electrophoretic ejection of Pontamine sky blue from the tip of the recording electrode $(-25 \mu \mathrm{A}$ constant current, 20-30 min). For acute studies, rats were killed by an overdose of anesthetic (chloral hydrate, additional $400 \mathrm{mg} / \mathrm{kg}$, i.p.), whereas for chronic studies, rats were killed by a lethal dose of anesthetic (sodium pentobarbital, $120 \mathrm{mg} / \mathrm{kg}$, i.p.). All rats were decapitated and their brains removed, fixed for at least $48 \mathrm{~h}(8 \% \mathrm{w} / \mathrm{v}$ paraformaldehyde in PBS), and cryoprotected $(25 \% \mathrm{w} / \mathrm{v}$ sucrose in PBS) until saturated. Brains were sectioned (60 $\mu \mathrm{m}$ coronal sections), mounted onto gelatin-chrom alum-coated slides, and stained with cresyl violet for histochemical verification of electrode and/or cannula sites. All histology was performed with reference to a stereotaxic atlas (Paxinos and Watson, 1986). Figure 1 provides a representation of the localization of bilateral injection sites within the ventral hippocampus.

Analysis. Electrophysiological analysis of DA and vHipp neuron activity was performed using custom-designed computer software (Neuroscope), whereas locomotor behavior was recorded using TruScan software (Coulbourn Instruments). All data are represented as the mean \pm SEM unless otherwise stated. All statistics were calculated using the SigmaStat software program (Systat Software, San Jose, CA).

Materials. MAM was purchased from Midwest Research Institute (Kansas City, MO). Ketamine $\mathrm{HCl}$ and xylazine were of United States Pharmacopeia (USP) grade and purchased from Phoenix Pharmaceutical (St. Joseph, MO) whereas pentobarbital sodium (USP) was obtained from Ovation Pharmaceuticals (Deerfield, IL). Chloral hydrate, NMDA, tetrodotoxin, gentamicin solution, dPBS, and D-amphetamine sulfate were all purchased from Sigma (St. Louis, MO). All other chemicals and reagents were of either analytical or laboratory grade and purchased from various suppliers.

\section{Results}

Rats that received GD17 saline injections ( $n=5$ rats, 45 neurons) exhibited an average of $1.15 \pm 0.05$ spontaneously active DA neurons per electrode track that fired at an average rate of $3.94 \pm$ $0.27 \mathrm{~Hz}$ with $26.4 \pm 3.6 \%$ of action potentials fired in bursts, consistent with previous findings in untreated rats (Floresco et al., 2003; Lodge and Grace, 2006a,b). Adult rats administered 

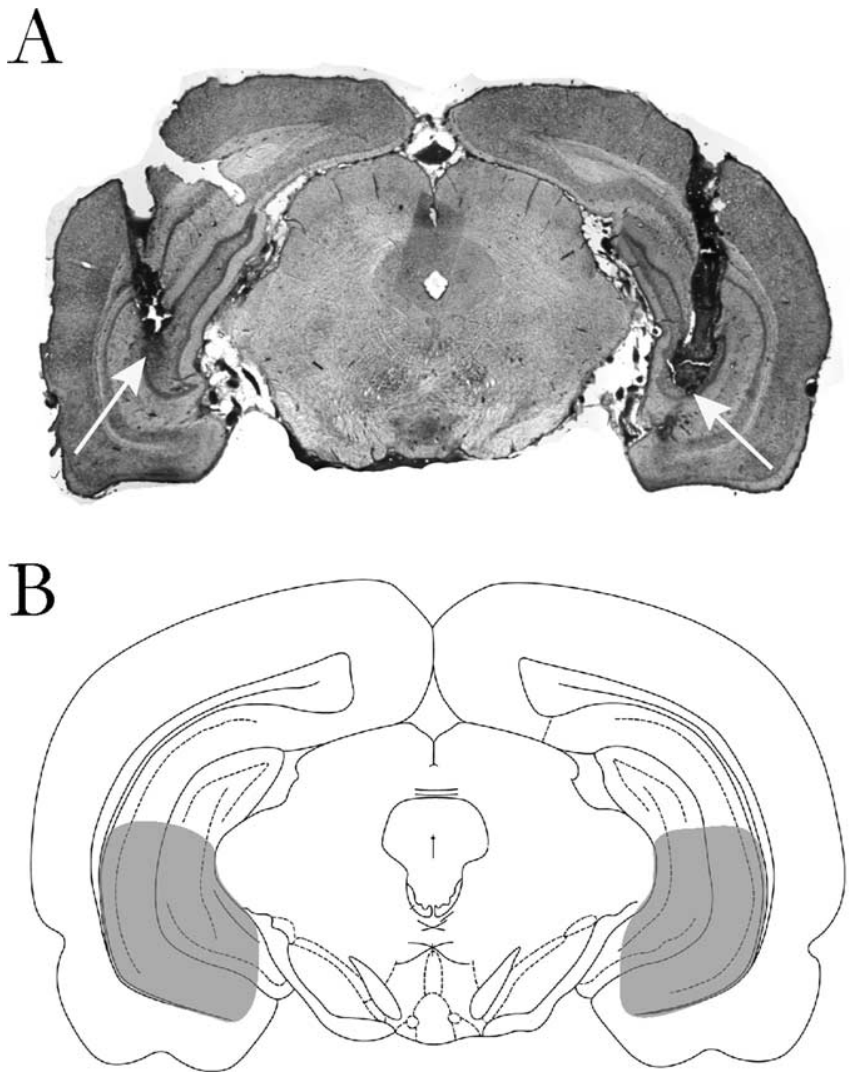

Figure 1. A, Representative section demonstrating the localization of bilateral cannulas placements in the ventral hippocampus. The injection sites are denoted by arrows. $B$, Schematic illustrating the target injection sites within the ventral hippocampus (shaded area). Plate adapted from Paxinos and Watson (1986).

MAM at GD17 ( $n=5$ rats, 63 neurons) exhibited significantly greater (approximately twofold) DA neuron population activity $(2.15 \pm 0.14$ cell/track; $p<0.05)$, without significant differences in average burst firing $(25.7 \pm 3.5 \%)$ or firing rate $(4.40 \pm 0.25$ $\mathrm{Hz}$ ) relative to control. In control animals ( $n=5$ rats, 62 neurons), the simultaneous NMDA-induced activation of the vHipp and PPTg resulted in a significant increase in DA neuron population activity $(2.07 \pm 0.12$ cells/track; $p<0.05)$ attributable to vHipp activation (Fig. $2 A$ ), and a significant increase in average burst firing $(46.7 \pm 3.8 \% ; p<0.05)$ attributable to PPTg activation (Fig. 2C) as reported previously (Lodge and Grace, 2006a). In contrast, in MAM-treated rats ( $n=5$ rats, 52 neurons), vHipp activation failed to further increase DA neuron population activity (Fig. $2 A)(1.86 \pm 0.04$ cells/track), likely attributable to a ceiling effect because it has been estimated that $\sim 50 \%$ of DA cells are quiescent at rest (Grace et al., 2007). Interestingly, PPTg afferent-induced burst firing remained intact $(39.3 \pm 3.7 \%$; $p<$ 0.05) (Fig. 2C).

Given evidence of hippocampal dysregulation in models of schizophrenia, we examined whether the activity of the vHipp was altered in MAM rats. Rats that received GD17 saline injections ( $n=4$ rats, 59 neurons) exhibited an average firing rate of $0.54 \pm 0.11 \mathrm{~Hz}$ with $42 \pm 3 \%$ of action potentials fired in bursts, an average within burst inter-spike interval (ISI) of $21.2 \pm 1.9 \mathrm{~ms}$ and an average of $2.4 \pm 0.1$ spikes per burst. In adult rats treated prenatally with MAM ( $n=4$ rats, 59 neurons), vHipp neurons exhibited significantly higher (more than twofold) average firing rates relative to control $(1.34 \pm 0.25 \mathrm{~Hz} ; p<0.05)$, without significant differences in any burst firing parameter (average

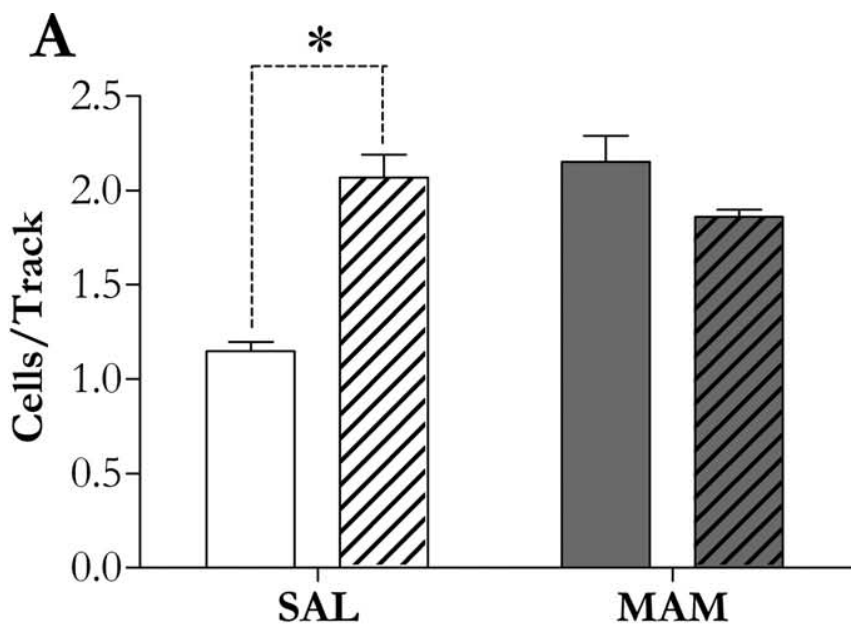

B
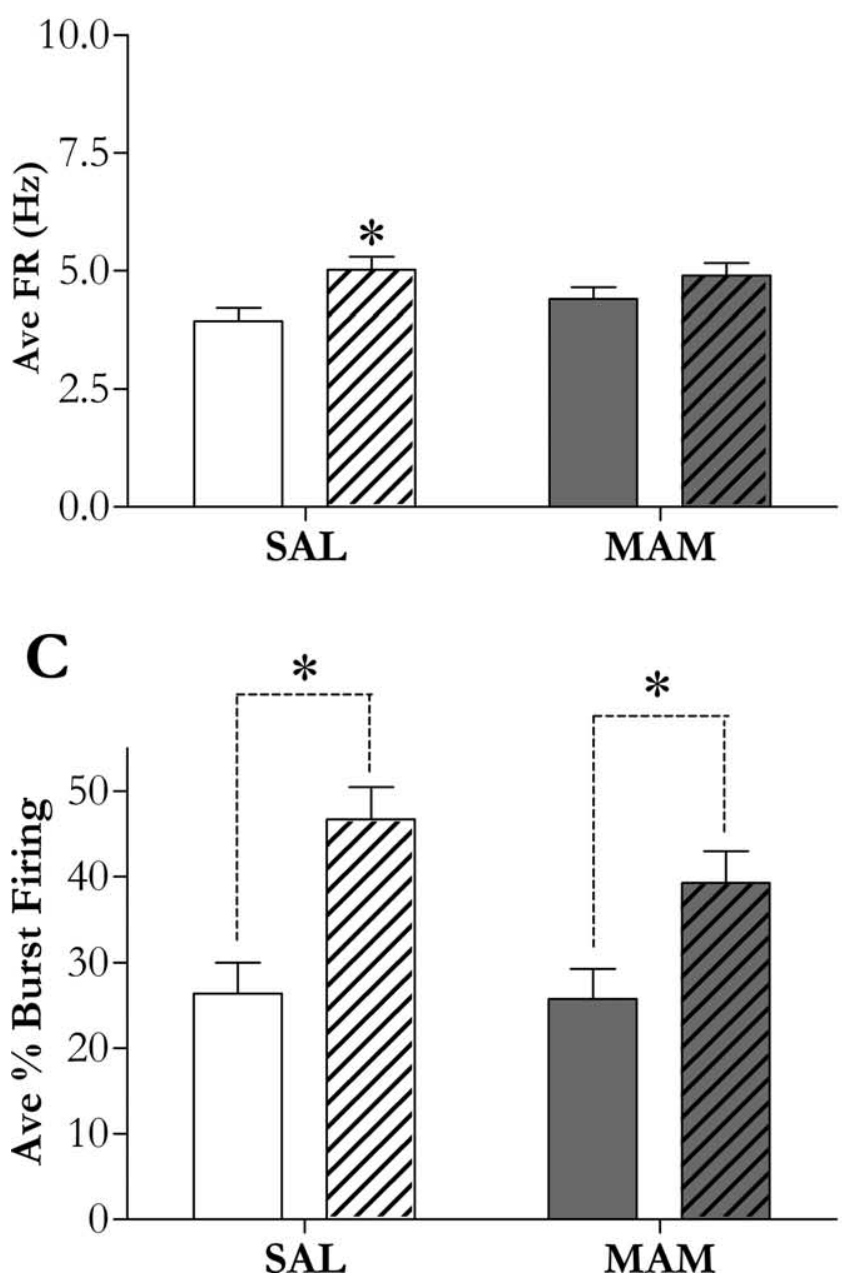

Figure 2. $A, C$, Afferent modulation of DA neuron activity states in MAM- and SAL-treated rats. In control rats (white bars), the simultaneous NMDA-induced activation of the vHipp and PPTg (patterned bars) resulted in a significant increase in DA neuron population activity $(\boldsymbol{A})$ attributable to vHipp activation (Lodge and Grace, 2006a); and a significant increase in average burst firing $(\boldsymbol{C})$ attributable to PPTg activation (Lodge and Grace, 2006a). In contrast, in MAMtreated rats (dark bars), vHipp activation failed to further increase DA neuron population activity $(\boldsymbol{A})$, whereas PPTg afferent-induced burst firing $(\boldsymbol{C})$ remained intact. $\boldsymbol{B}$, The effect of NMDAinduced afferent activation on average firing rate. An asterisk represents a statistically significant difference from control ( $p<0.05$, one-way ANOVA, Student-Newman-Keuls posthoc or, if data failed tests for normality and/or equal variance, a Kruskal-Wallis one-way ANOVA on Ranks, Dunn's post hoc; $n=5$ rats/group). SAL, Saline-treated. 
burst firing, $38 \pm 3 \%$; average within burst ISI, $24.7 \pm 2.7 \mathrm{~ms}$; average spikes per burst: $2.7 \pm 0.2$ ).

Given the role of the hippocampus in the regulation of DA neuron population activity, we examined whether the observed hippocampal overactivity may be responsible for the aberrant DA neuron population activity. Intra-vHipp administration of the sodium channel blocked TTX to MAM-treated rats $(n=5$ rats, 33 neurons) normalized the population activity to a level not significantly different from control $(0.99 \pm 0.12$ cells/track) (Fig. $3 A$ ). Importantly, blockade of hippocampal transmission with TTX did not significantly alter any parameter of DA neuron activity in control rats ( $n=5$ rats, 41 neurons; population activity, $1.17 \pm 0.09$ cells/track; firing rate, $3.83 \pm 0.26 \mathrm{~Hz}$; burst firing, $26.9 \pm 3.9 \%$ ) (Fig. $3 A-C)$, nor did it affect firing rate $(4.20 \pm 0.32$ $\mathrm{Hz})$ or burst firing $(27.5 \pm 4.8 \%)$ in MAM-treated rats (Fig. $3 B, C)$. It should be noted that no significant change in cells/track within animals across time was observed, suggesting that the effect of TTX was not associated with diffusion into adjacent structures. Furthermore, it should be noted that this is a well established technique used to examine the involvement of the vHipp in numerous behavioral (Ambrogi Lorenzini et al., 1997; Zhang et al., 2002; Degroot and Treit, 2004), neurochemical (Legault and Wise, 1999; Peterschmitt et al., 2005), and electrophysiological (Floresco et al., 2001) studies.

Given the previous literature demonstrating an increased behavioral responsivity to amphetamine in animal models of schizophrenia, including MAM rats (Flagstad et al., 2004; Moore et al., 2006), we suggest that this may be attributed to the enhanced baseline DA neuron population activity secondary to vHipp hyperactivity. Consistent with previous observations (Flagstad et al., 2004; Moore et al., 2006), MAM-treated rats displayed a significantly enhanced locomotor response $(\sim 15 \%$ increase above that produced in controls; $p<0.05)$ to low dose $(0.5$ $\mathrm{mg} / \mathrm{kg}$, i.p.) D-amphetamine administration (Fig. $4 A$ ). Furthermore, whereas bilateral hippocampal inactivation had no significant effect on amphetamine-induced locomotor activity in control animals (Fig. 4B), it significantly reduced the increased psychostimulant-induced locomotion observed in MAM rats (Fig. 4C). Taken as a whole, these data suggest that, in MAM rats, a pathologically increased DA neuron population activity and increased responsivity to amphetamine are attributable to basal hippocampal hyperactivity.

\section{Discussion}

The data presented here demonstrate that MAM-treated rats display a pathologically enhanced DA neuron drive in the form of an increase in DA neuron population activity. Moreover, this seems to be associated with an increase in the spontaneous firing rate of vHipp neurons unrelated to changes in patterned activity at the single cell level. Furthermore, we suggest that the aberrant DA neuron activity is attributed to the increased vHipp activity because intra-vHipp TTX administration normalizes both the augmented DA neuron activity and the behavioral hyperresponsivity to amphetamine. It is important to note that TTX administration did not eliminate the response to amphetamine, but instead restored it to the level found in controls.

An association between hippocampal activity and ascending DA function has been suggested previously (Legault and Wise, 1999; Floresco et al., 2001, 2003; Lodge and Grace, 2006a). Thus, the vHipp can modulate DA neuron population activity via a multisynaptic (vHipp-NAc-VP-VTA) pathway (Floresco et al., 2001, 2003). Furthermore, we have demonstrated previously that the hippocampal modulation of DA neuron population activity is
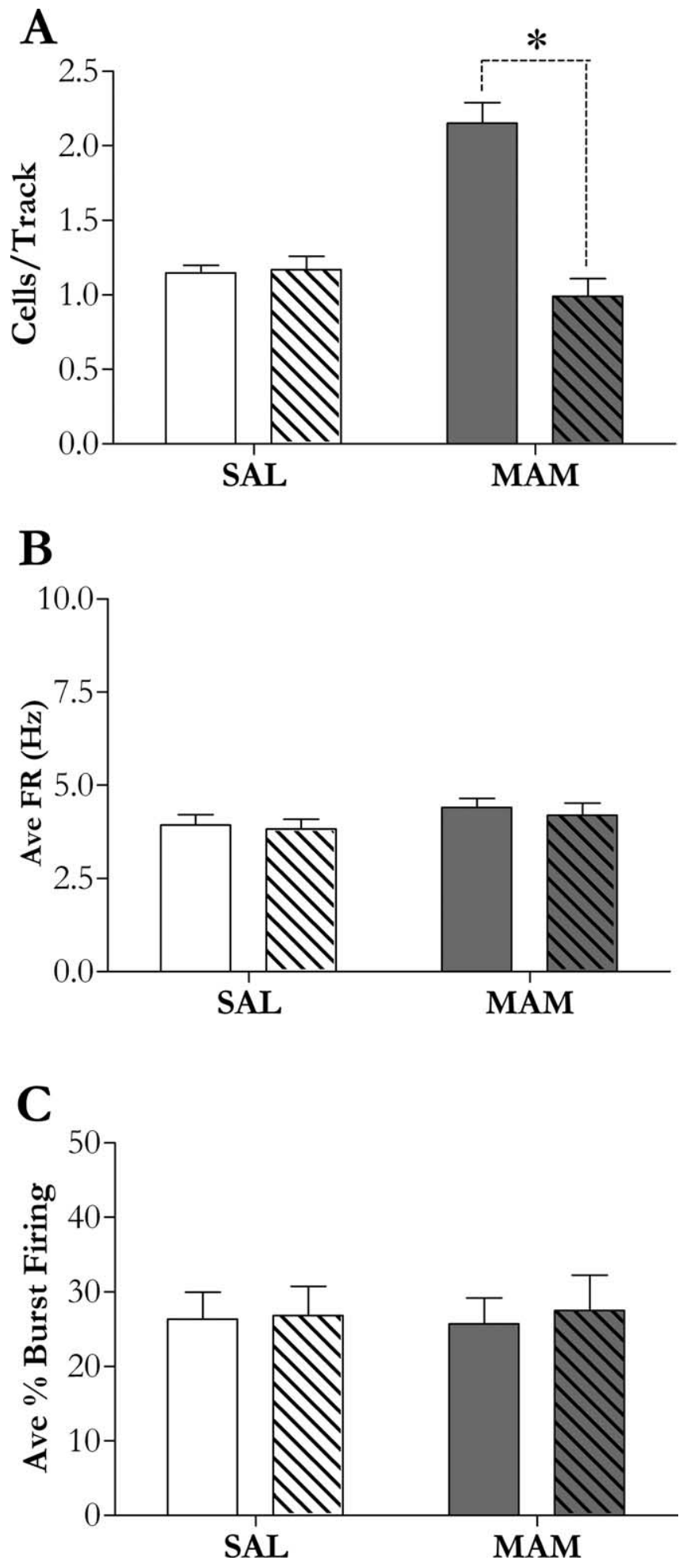

Figure 3. A-C, Inactivation of the vHipp by TTX ( $1 \mu \mathrm{m}$; patterned bars) normalizes the aberrant increase in DA neuron population activity in MAM rats $(\boldsymbol{A})$, although has no observable effect in control rats (white bars; $A-C$ ) or on any other DA neuron activity state in MAM rats (dark bars; $\boldsymbol{B}, \boldsymbol{C})$. An asterisk represents a significant difference between vehicle and $v$ Hipp inactivated rats ( $p<0.05$, one-way ANOVA, Student-Newman-Keuls post hoc or, if data failed tests for normality and/or equal variance, a Kruskal-Wallis one-way ANOVA on Ranks, Dunn's post hoc; $n=5$ rats/group). SAL, Saline-treated.

not simply associated with the tonic release of DA in forebrain regions, but also represents a recruitable pool of DA neurons that can be further modulated by excitatory inputs to induce a graded 
A

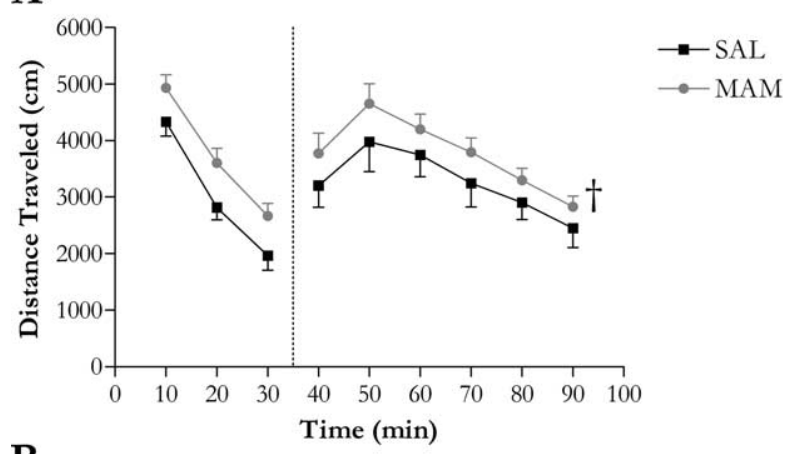

B
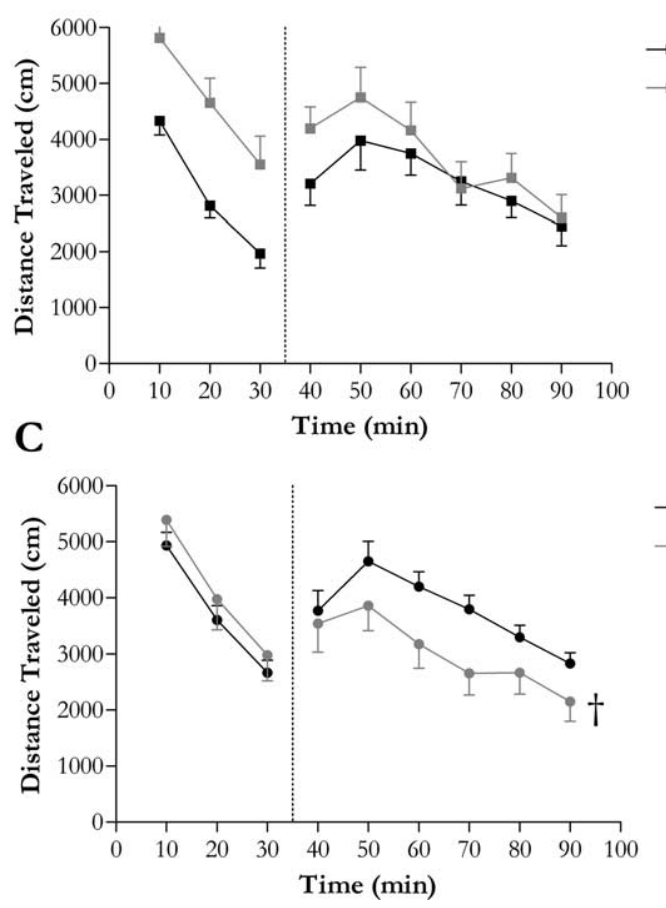

Figure 4. Bilateral vHipp inactivation by TTX $(1 \mu \mathrm{M})$ normalizes the aberrant locomotor response to $\mathrm{D}$-amphetamine $(0.5 \mathrm{mg} / \mathrm{kg}$ i.p.) observed in MAM rats. A, MAM-treated rats display an increased response to D-amphetamine administration compared with saline-treated rats. $\boldsymbol{B}$, C, Inactivation of the vHipp by TTX significantly attenuates the locomotor response to $D$-amphetamine in MAM rats $(\boldsymbol{C})$ although has no significant effect on psychostimulant-induced locomotion in saline-treated (SAL) rats $(\boldsymbol{B})$. ${ }^{\dagger}$ Significant difference from control $(p<0.05$, two-way ANOVA, Tukey's post hoc; $n=9-17$ rats/group).

phasic response (Lodge and Grace, 2006a). Therefore, increases in vHipp activity will lead to an augmentation of DA system responsivity. We now demonstrate that a pathologically enhanced hippocampal activity can result in aberrant DA neuron signaling in a verified model of schizophrenia. Specifically, we demonstrate that MAM-treated rats display a significantly higher number of spontaneously active DA neurons compared with control rats. Moreover, although PPTg afferent-induced burst firing remained intact in MAM rats, additional activation of the vHipp failed to induce additional increases in DA neuron population activity in this model. We demonstrated previously the importance of the hippocampal input in regulating not only the tonic DA signal, but also the number of DA neurons capable of conveying the phasic DA signal (Lodge and Grace, 2006a). As such, the failure of hippocampal activation to increase DA neuron population activity reflects a loss of a process critical for regulating DA neuron output in MAM rats.
Given evidence of hippocampal dysregulation in schizophrenia, we examined whether activity within the ventral hippocampus was altered in MAM-treated rats. Thus, the activity of vHipp neurons is enhanced in MAM rats, expressed as a significantly greater baseline firing rate with no significant change in patterned activity at the single cell level. Because the hippocampus potently modulates DA neuron population activity, this hippocampal hyperactivity may be responsible for the aberrant DA neuron population activity in MAM-treated rats. Indeed, TTX inactivation of the vHipp normalized the pathologically enhanced DA neuron population activity to a level consistent with that routinely observed in control animals. This manipulation had no significant effect on any other parameter of DA neuron activity in MAM rats nor did it have any observable effects on DA neuron activity in control animals. This lack of effect is consistent with the way the hippocampus modulates DA neuron population activity, (i.e., via a polysynaptic disinhibition of VTA activity) (Floresco et al., 2001,2003). More specifically, given that the ventral pallidum has a high degree of spontaneous activity $(>10 \mathrm{~Hz}$ ) (Johnson and Napier, 1997), whereas cells of the NAc are largely quiescent (Mulder et al., 1997) in the anesthetized rat, it is not surprising that inhibition of hippocampal activity has little or no effect on baseline DA cell activity.

There is a significant literature demonstrating an increased responsivity to psychostimulants in both human schizophrenia patients (Laruelle et al., 1996; Breier et al., 1997) and animal models of schizophrenia, including MAM rats (Flagstad et al., 2004; Moore et al., 2006). Moreover, vHipp activation has been shown to increase the behavioral response to amphetamine in normal rats (White et al., 2006). Therefore, we propose that the source of amphetamine hyper-responsivity may be attributed to vHipp-induced enhancement of baseline DA neuron population activity. Whereas bilateral hippocampal inactivation had no significant effect on amphetamine-induced locomotor activity in control animals (consistent with the lack of effect observed on DA cell activity), it significantly reduced the augmented psychostimulant-induced locomotion observed in MAM rats. Together, these data suggest that, in MAM rats, a pathologically increased DA neuron population activity and increased responsivity to amphetamine are attributable to basal hippocampal hyperactivity, although we have not tested whether the hyperactivity is present in the prepubertal animal, in which amphetamine fails to cause hyperactivation in the MAM model. Moreover, given the results of the present study, we propose that the hippocampal dysfunction present in schizophrenia patients is the basis for the hyper-responsive dopamine system proposed to underlie psychosis in this disorder.

We suggested previously that disruption of hippocampal projections to the NAc may be a pathophysiological factor in schizophrenia (Grace and Moore, 1998; O’Donnell and Grace, 1998; Goto and Grace, 2005). The model advanced here is consistent with hippocampal dysfunction observed in schizophrenia patients (Saykin et al., 1991; Harrison, 1999; Shenton et al., 2001) and functional imaging studies show abnormally high activity both during resting states (Nordahl et al., 1996; Heckers et al., 1998; Lahti et al., 2006) and during task performance (Medoff et al., 2001; Meyer-Lindenberg et al., 2001; Weiss et al., 2006). In addition, an increase in hippocampal volume has been reported in patients at the time of first psychotic break (Pantelis et al., 2003), suggestive of hyperactivation within this structure. Furthermore, hippocampal hyperactivity is proposed to underlie the abnormal thought processes, hallucinations, and delusions in this disorder (Krieckhaus et al., 1992; Venables, 1992). Finally, it 
is well known that temporal lobe epilepsy, a type of hyperactivity of the hippocampus, has been associated with schizophrenia symptoms in humans (Ounsted and Lindsay, 1981). Together, these data are consistent with the model derived from the present study, in which a hyperactive vHipp drives a DA hyperfunction. However, whether this hyperactivity has its origin as a result of pathology within the ventral hippocampus, or is driven abnormally by another region, is not known at this time.

At face value, the hippocampal hyperactivity model of schizophrenia may appear to be inconsistent with the neonatal ventral hippocampal lesion (NVHL) model (Lipska et al., 1993). However, we suggest that both models may actually be producing a similar pathology, but via different means. Thus, Swerdlow et al. (2001) have demonstrated that the enhanced behavioral responsivity to amphetamine in the NVHL model is attenuated with extensive lesions encompassing the entire dorsal and ventral regions of the hippocampus. On this basis, it has been suggested that the behavioral abnormalities in the NVHL model reflect, at least in part, aberrant function within spared elements of the hippocampal complex (Swerdlow et al., 2001). Therefore, although this increase in hippocampal activity has not been demonstrated in the NVHL model, it is possible that in both models the enhanced response to amphetamine derives from a hyperactivity within hippocampal tissue.

The present study demonstrates that the pathological increase in tonic DA transmission and aberrant responsivity to psychomotor stimulants observed in MAM rats is likely attributable to hyperactivity within the ventral hippocampus. Moreover, we propose that the hippocampal dysfunction consistently observed in schizophrenia patients is the basis for the dopamine dysregulation in this disorder. We are of course aware that the administration of a toxin to a developing rat is not an accurate recapitulation of the etiology of schizophrenia in humans, nor is the presence of simple deficits in sensory gating and executive function a necessary parallel to the complex cognitive and affective deficits distinctive of this disorder. Nonetheless, we posit that at the core of this disorder is a disruption of systems interactions that can be modeled in animals, but when placed in the context of complex human brain and behavioral patterns, yields the complex pattern of psychopathology recognized as schizophrenia. Such an understanding of the functional interactions among these systems and how disruption within these circuits affects information processing is central to gaining a better understanding of disease pathophysiology and developing better pharmacotherapeutic agents.

\section{References}

Abi-Dargham A (2004) Do we still believe in the dopamine hypothesis? New data bring new evidence. Int J Neuropsychopharmacol 7:S1-S5.

Ambrogi Lorenzini CG, Baldi E, Bucherelli C, Sacchetti B, Tassoni G (1997) Role of ventral hippocampus in acquisition, consolidation and retrieval of rat's passive avoidance response memory trace. Brain Res 768:242-248.

Berridge KC, Robinson TE (1998) What is the role of dopamine in reward: hedonic impact, reward learning, or incentive salience? Brain Res Rev 28:309-369.

Breier A, Su TP, Saunders R, Carson RE, Kolachana BS, de Bartolomeis A, Weinberger DR, Weisenfeld N, Malhotra AK, Eckelman WC, Pickar D (1997) Schizophrenia is associated with elevated amphetamine-induced synaptic dopamine concentrations: evidence from a novel positron emission tomography method. Proc Natl Acad Sci USA 94:2569-2574.

Chergui K, Akaoka H, Charlety PJ, Saunier CF, Buda M, Chouvet G (1994) Subthalamic nucleus modulates burst firing of nigral dopamine neurones via NMDA receptors. NeuroReport 5:1185-1188.

Degroot A, Treit D (2004) Anxiety is functionally segregated within the septo-hippocampal system. Brain Res 1001:60-71.
Flagstad P, Mork A, Glenthoj BY, van Beek J, Michael-Titus AT, Didriksen M (2004) Disruption of neurogenesis on gestational day 17 in the rat causes behavioral changes relevant to positive and negative schizophrenia symptoms and alters amphetamine-induced dopamine release in nucleus accumbens. Neuropsychopharmacology 29:2052-2064.

Floresco SB, Todd CL, Grace AA (2001) Glutamatergic afferents from the hippocampus to the nucleus accumbens regulate activity of ventral tegmental area dopamine neurons. J Neurosci 21:4915-4922.

Floresco SB, West AR, Ash B, Moore H, Grace AA (2003) Afferent modulation of dopamine neuron firing differentially regulates tonic and phasic dopamine transmission. Nat Neurosci 6:968-973.

Goto Y, Grace AA (2005) Dopamine-dependent interactions between limbic and prefrontal cortical plasticity in the nucleus accumbens: disruption by cocaine sensitization. Neuron 47:255-266.

Goto Y, Grace AA (2006) Alterations in medial prefrontal cortical activity and plasticity in rats with disruption of cortical development. Biol Psychiatry 60:1259-1267.

Gourevitch R, Rocher C, Le Pen G, Krebs MO, Jay TM (2004) Working memory deficits in adult rats after prenatal disruption of neurogenesis. Behav Pharmacol 15:287-292.

Grace AA (1991) Phasic versus tonic dopamine release and the modulation of dopamine system responsivity: a hypothesis for the etiology of schizophrenia. Neuroscience 41:1-24.

Grace AA (2000) Gating of information flow within the limbic system and the pathophysiology of schizophrenia. Brain Res Rev 31:330-341.

Grace AA, Bunney BS (1983) Intracellular and extracellular electrophysiology of nigral dopaminergic neurons. I. Identification and characterization. Neuroscience 10:301-315.

Grace AA, Moore H (1998) Regulation of information flow in the nucleus accumbens: a model for the pathophysiology of schizophrenia. In: Origins and development of schizophrenia: advances in experimental psychopathology (Lenzenweger MF, Dworkin RH, eds), pp 123-157. Washington, DC: American Psychological Association.

Grace AA, Floresco SB, Goto Y, Lodge DJ (2007) Regulation of firing of dopaminergic neurons and control of goal-directed behaviors. Trends Neurosci 30:220-227.

Harrison PJ (1999) The neuropathology of schizophrenia. A critical review of the data and their interpretation. Brain 122:593-624.

Harrison PJ (2004) The hippocampus in schizophrenia: a review of the neuropathological evidence and its pathophysiological implications. Psychopharmacology 174:151-162.

Heckers S, Rauch SL, Goff D, Savage CR, Schacter DL, Fischman AJ, Alpert NM (1998) Impaired recruitment of the hippocampus during conscious recollection in schizophrenia. Nat Neurosci 1:318-323.

Johnson PI, Napier TC (1997) GABA- and glutamate-evoked responses in the rat ventral pallidum are modulated by dopamine. Eur J Neurosci 9:1397-1406.

Krieckhaus EE, Donahoe JW, Morgan MA (1992) Paranoid schizophrenia may be caused by dopamine hyperactivity of CA1 hippocampus. Biol Psychiatry 31:560-570.

Lahti AC, Weiler MA, Holcomb HH, Tamminga CA, Carpenter WT, McMahon R (2006) Correlations between rCBF and symptoms in two independent cohorts of drug-free patients with schizophrenia. Neuropsychopharmacology 31:221-230.

Laruelle M, Abi-Dargham A (1999) Dopamine as the wind of psychotic fire: new evidence from brain imaging studies. J Psychopharmacol 13:358-371.

Laruelle M, Abi-Dargham A, Van Dyck CH, Gil R, D’Souza CD, Erdos J, McCance E, Rosenblatt W, Fingado C, Zoghbi SS, Baldwin RM, Seibyl JP, Krystal JH, Charney DS, Innis RB (1996) Single photon emission computerized tomography imaging of amphetamine-induced dopamine release in drug-free schizophrenic subjects. Proc Natl Acad Sci USA 93:9235-9240.

Legault M, Wise RA (1999) Injections of N-methyl-D-aspartate into the ventral hippocampus increase extracellular dopamine in the ventral tegmental area and nucleus accumbens. Synapse 31:241-249.

Lipska BK, Jaskiw GE, Weinberger DR (1993) Postpubertal emergence of hyperresponsiveness to stress and to amphetamine after neonatal excitotoxic hippocampal damage: a potential animal model of schizophrenia. Neuropsychopharmacology 9:67-75.

Lodge DJ, Grace AA (2006a) The hippocampus modulates dopamine neu- 
ron responsivity by regulating the intensity of phasic neuron activation. Neuropsychopharmacology 31:1356-1361.

Lodge DJ, Grace AA (2006b) The laterodorsal tegmentum is essential for burst firing of ventral tegmental area dopamine neurons. Proc Natl Acad Sci USA 103:5167-5172.

Medoff DR, Holcomb HH, Lahti AC, Tamminga CA (2001) Probing the human hippocampus using rCBF: contrasts in schizophrenia. Hippocampus 11:543-550.

Meyer-Lindenberg A, Poline JB, Kohn PD, Holt JL, Egan MF, Weinberger DR, Berman KF (2001) Evidence for abnormal cortical functional connectivity during working memory in schizophrenia. Am J Psychiatry 158:1809-1817.

Moore H, Jentsch JD, Ghajarnia M, Geyer MA, Grace AA (2006) A neurobehavioral systems analysis of adult rats exposed to methylazoxymethanol acetate on E17: implications for the neuropathology of schizophrenia. Biol Psychiatry 60:253-264.

Mulder AB, Arts MP, Lopes Da Silva FH (1997) Short- and long-term plasticity of the hippocampus to nucleus accumbens and prefrontal cortex pathways in the rat, in vivo. Eur J Neurosci 9:1603-1611.

Nordahl TE, Kusubov N, Carter C, Salamat S, Cummings AM, O'ShoraCelaya L, Eberling J, Robertson L, Huesman RH, Jagust W, Budinger TF (1996) Temporal lobe metabolic differences in medication-free outpatients with schizophrenia via the PET-600. Neuropsychopharmacology 15:541-554.

O'Donnell P, Grace AA（1998) Phencyclidine interferes with the hippocampal gating of nucleus accumbens neuronal activity in vivo. Neuroscience 87:823-830.

Ounsted C, Lindsay J (1981) Epilepsy and psychiatry (Reynolds EH, Trinble MR, eds). Edinburgh: Churchill Livingstone.

Pantelis C, Velakoulis D, McGorry PD, Wood SJ, Suckling J, Phillips LJ, Yung AR, Bullmore ET, Brewer W, Soulsby B, Desmond P, McGuire PK (2003) Neuroanatomical abnormalities before and after onset of psychosis: a cross-sectional and longitudinal MRI comparison. Lancet 361:281-288.
Paxinos G, Watson C (1986) The rat brain in stereotaxic coordinates. Sydney: Academic Australia.

Peterschmitt Y, Hoeltzel A, Louilot A (2005) Striatal dopaminergic responses observed in latent inhibition are dependent on the hippocampal ventral subicular region. Eur J Neurosci 22:2059-2068.

Saykin AJ, Gur RC, Gur RE, Mozley PD, Mozley LH, Resnick SM, Kester DB, Stafiniak P (1991) Neuropsychological function in schizophrenia. Selective impairment in memory and learning. Arch Gen Psychiatry 48:618-624.

Schultz W (1998) Predictive reward signal of dopamine neurons. J Neurophysiol 80:1-27.

Shenton ME, Dickey CC, Frumin M, McCarley RW (2001) A review of MRI findings in schizophrenia. Schizophr Res 49:1-52.

Swerdlow NR, Halim N, Hanlon FM, Platten A, Auerbach PP (2001) Lesion size and amphetamine hyperlocomotion after neonatal ventral hippocampal lesions: more is less. Brain Res Bull 55:71-77.

Talamini LM, Koch T, Ter Horst GJ, Korf J (1998) Methylazoxymethanol acetate-induced abnormalities in the entorhinal cortex of the rat; parallels with morphological findings in schizophrenia. Brain Res 789:293-306.

Talamini LM, Ellenbroek B, Koch T, Korf J (2000) Impaired sensory gating and attention in rats with developmental abnormalities of the mesocortex. Implications for schizophrenia. Ann NY Acad Sci 911:486-494.

Venables PH (1992) Hippocampal function and schizophrenia. Experimental psychological evidence. Ann NY Acad Sci 658:111-127.

Weiss AP, Goff D, Schacter DL, Ditman T, Freudenreich O, Henderson D, Heckers S (2006) Fronto-hippocampal function during temporal context monitoring in schizophrenia. Biol Psychiatry 60:1268-1277.

White IM, Whitaker C, White W (2006) Amphetamine-induced hyperlocomotion in rats: hippocampal modulation of the nucleus accumbens. Hippocampus 16:596-603.

Zhang WN, Bast T, Feldon J (2002) Prepulse inhibition in rats with temporary inhibition/inactivation of ventral or dorsal hippocampus. Pharmacol Biochem Behav 73:929-940. 